\title{
ELABORAÇÃO DE COOKIES COM ADIÇÃO DE FARINHA DE CASCA DE BATATA: ANÁLISE FÍSICO-QUÍMICA E SENSORIAL
}

\author{
Rosa PA*, Santos MMRS ${ }^{* *}$, Candido CJ ${ }^{* * *}$, Schwarz K ${ }^{* * *}$, Santos EF ${ }^{* * * *}$, Novello D ${ }^{* * * * *}$
}

\begin{abstract}
Resumo
No presente estudo teve-se como objetivo elaborar cookies com adição de farinha de casca de batata (FCB) e avaliar a sua aceitabilidade sensorial, bem como determinar a composição físico-química do produto padrão e do produto com maior teor de FCB, e se a aceitação é semelhante a do produto padrão. Foram elaboradas as seguintes formulações de cookies: F1 (padrão - 0\%) e as demais adicionadas de 6\% (F2), 8,5\% (F3), 11\% (F4) e 13,5\% (F5) de FCB. Participaram da avaliação sensorial 62 provadores não treinados, de ambos os gêneros, com idade entre 18 e 55 anos. Verificou-se que para os atributos de aparência, textura, cor, aceitação global e intenção de compra houve uma redução das notas a partir da adição de 6\% de FCB (F2) (p<0,05). Para o aroma e sabor, a aceitabilidade foi signifi-

\footnotetext{
* Graduada em Nutrição pela Universidade Estadual do Centro-Oeste; patricia.amancio94@hotmail.com

** Mestre e doutoranda em Saúde e Desenvolvimento na Região Centro-Oeste pela Universidade Federal de Mato Grosso do Sul; Graduada em Nutrição pela Universidade Federal de Mato Grosso do Sul; mirellymarques@hotmail.com

${ }^{* * *}$ Mestre em Saúde e Desenvolvimento na Região Centro-Oeste pela Universidade Federal de Mato Grosso do Sul; Graduada em Farmácia pela Universidade Federal de Mato Grosso do Sul; cahjordao@gmail.com.br

${ }^{* * * *}$ Doutora em Ciências pela Universidade de São Paulo; Mestre em Agronomia pela Universidade Estadual do Centro-Oeste; kelinschwarz@hotmail.com

${ }_{* * * * *}^{*}$ Doutora em Ciências da Cirurgia pela Universidade Estadual de Campinas; Mestre em Alimentos e Nutrição pela Universidade Estadual de Campinas; elisvania@gmail.com

${ }_{* * * * * *}$ Doutora em Tecnologia de Alimentos pela Universidade Estadual de Campinas; Mestre em Ciências Veterinárias pela Universidade Federal do Paraná; Professora do Curso de Nutrição na Universidade Estadual do Centro-Oeste do Paraná; nutridai@gmail.com
} 
cativamente menor para as formulações F4 e F5. Menores teores de cinzas, proteínas e fibra alimentar e maiores de lipídios foram constatados em F1 quando comparada à F3. Conclui-se que um nível de adição de até 8,5\% de FCB em cookies foi bem aceito pelos provadores, obtendo-se aceitação sensorial semelhante ao produto padrão e com boas expectativas de comercialização.

Palavras-chave: Aceitabilidade. Aproveitamento. Fibras.

\title{
Elaboration of cookies with addition of potato skin flour: physico-chemical and sensory analysis
}

\begin{abstract}
This study aimed to elaborate cookies with added potato skin flour (PSF) and evaluate their sensory acceptability, and to determine the physico-chemical composition of the standard product and of the product with more PSF content, and if the acceptance is similar to the standard product. The cookies formulations produced were: F1 (standard - 0\%) and the others added 6\% (F2), 8.5\% (F3), 11\% (F4) and 13.5\% (F5) of PSF. Participated in the sensory evaluation 62 untrained panelists, of both genders, aged 18 to 55 years. It was found that for the appearance attributes, texture, color, overall acceptability, and purchase intention there was a reduction of the notes from the addition of 6\% PSF (F2) ( $p<0.05)$. However, for aroma and taste the acceptability was significantly lower for formulations F4 and F5. Lower ash, protein and dietary fiber and higher in lipids content were observed in F1 compared to F3. It is concluded that an addition level of $8.5 \%$ to PSF in cookies was well accepted by the panelists, and had sensory acceptance similar to standard product with good market expectation.
\end{abstract}

Keywords: Acceptability. Harnessing. Fibers.

\section{INTRODUÇÃO}

O Brasil é um dos países que mais desperdiça alimentos no mundo, podendo chegar a 26 milhões de toneladas/ano. No caso específico das hortaliças, o Brasil apresenta uma produção de aproximadamente 17 milhões/ano, dos quais sete milhões não chegam aos consumidores em razão de serem descartados em etapas de produção e armazenamento. ${ }^{1,2}$ Em vista disso, os subprodutos alimentícios poderiam ser utilizados como ingredientes em preparações habitualmente consumidas, colaborando para reduzir o desperdício e para aumentar o valor nutricional dos produtos, isso porque, em muitos casos, alimentos não convencionais como talos, cascas e folhas podem conter mais nutrientes que as partes nobres das frutas e vegetais. ${ }^{2}$

A batata inglesa (Solanum tuberosum L.) é considerada uma das principais culturas alimentícias brasileiras. Sua comercialização ocorre, principalmente, na forma in natura e apresenta uma produção estimada de 3,6 milhões de toneladas/ano. ${ }^{3,4} \mathrm{Na}$ última década, o consumo de batata in natura tem diminuído bastante, porém houve um aumento considerável de consumo em sua forma processada. Após o processamento da batata, uma grande quantidade de resíduos é gerada na forma 
de cascas e aparas, o que pode causar problemas de manuseio e armazenamento. Apesar desse con-

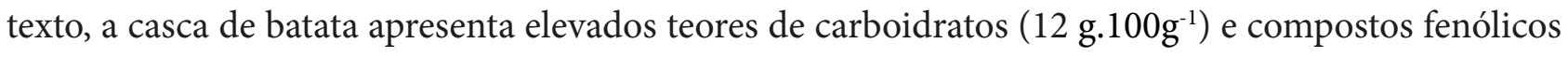
(25-125 mg. $\left.100 \mathrm{~g}^{-1}\right)$, além de conter proteínas $\left(2,56 \mathrm{~g} \cdot 100 \mathrm{~g}^{-1}\right)$ e lipídios $\left(0,1 \mathrm{~g} \cdot 100 \mathrm{~g}^{-1}\right) .^{5}$ Assim, a casca de batata torna-se um potencial ingrediente para ser adicionado em novos produtos alimentícios. Sabendo-se disso, muitos subprodutos de frutas e vegetais já estão sendo adicionados e avaliados em várias preparações como, por exemplo, biscoitos e bolos, que têm obtido boa aceitabilidade sensorial. ${ }^{6}$

Os cookies são conceituados como biscoitos adocicados, consumidos por todas as idades, além de apresentarem um shelf life relativamente longo. Destaca-se o elevado consumo de biscoitos na fase adulta e na adolescência, estando entre os 20 alimentos com maior prevalência de ingestão no País (15,9\%). ${ }^{4}$ A indústria de biscoitos no Brasil registrou um crescimento de 7,1\% em 2015 comparado a 2014. A produção anual foi de 1,7 milhão de toneladas, com consumo per capita de 8,5 kg/ano e

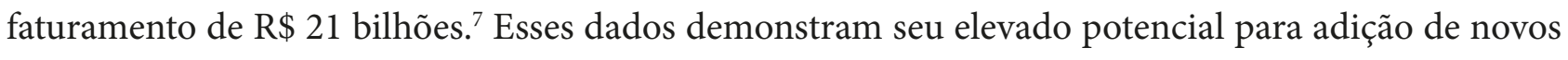
ingredientes e possibilidade de comercialização.

Para que um novo produto seja desenvolvido e comercializado é necessário o uso de testes científicos que comprovem sua boa aceitação e adequada padronização quanto aos aspectos legais. Uma ferramenta muito utilizada para avaliar a aceitação de um produto é a análise sensorial, a qual possibilita um amplo estudo das propriedades do alimento, além de identificar as expectativas dos consumidores em relação ao novo produto. ${ }^{8}$ Da mesma forma, a avaliação das características físico-químicas fornece a composição nutricional do alimento, o que colabora para a escolha de alimentos mais saudáveis pela população, além de ser importante para o controle da qualidade nutricional do produto, que deve respeitar a regulamentação específica.

O objetivo com este trabalho foi elaborar cookies com adição de farinha de casca de batata (FCB) e avaliar a sua aceitabilidade sensorial. Também se objetivou determinar a composição físico-química do produto padrão e do produto com maior teor de FCB e aceitação semelhante ao padrão.

\section{MATERIAL E MÉTODOS}

\subsection{AQUISIÇÃO DA MATÉRIA-PRIMA}

Os ingredientes foram adquiridos em supermercados do Município de Guarapuava, PR. Foram utilizadas batatas (Solanum tuberosum L.) com melhor aspecto visual, superfície lisa e sem imperfeições.

\subsection{PREPARAÇÃO DA FARINHA}

Quinze quilos de batatas foram higienizados em água corrente potável, sanitizados (mergulhados em solução de hipoclorito de sódio por 15 minutos) e novamente higienizados em água cor- 
rente. As cascas foram extraídas de forma manual (espessura aproximada de 1,5 $\mathrm{mm}$ ) e picadas, posteriormente foram submetidas à secagem em estufa com circulação de ar $\left(65^{\circ} \mathrm{C}\right)$ por 24 horas. Depois de desidratadas, permaneceram em temperatura ambiente $\left(22^{\circ} \mathrm{C}\right)$ até total resfriamento. Logo após, foram trituradas em liquidificador doméstico e peneiradas em peneira com abertura de $32 \mathrm{mesh} / \mathrm{Ty}$ ler, até a obtenção da FCB, com um rendimento de $568 \mathrm{~g}$.

\subsection{FORMULAÇÕES}

Foram elaboradas cinco formulações de cookies (Tabela 1). A porcentagem de adição dos ingredientes foi definida por meio de testes sensoriais preliminares realizados com o produto.

Tabela 1 - Ingredientes das formulações de cookies adicionados de farinha de casca de batata

\begin{tabular}{lrrrrr}
\hline \multicolumn{1}{c}{ Ingredientes } & F1 (\%) & F2 (\%) & F3 (\%) & F4 (\%) & F5 (\%) \\
\hline Farinha de trigo & 32,0 & 26,0 & 23,5 & 21,0 & 18,5 \\
Chocolate meio amargo & 23,1 & 23,1 & 23,1 & 23,1 & 23,1 \\
Açúcar refinado & 18,6 & 18,6 & 18,6 & 18,6 & 18,6 \\
Ovos & 18,1 & 18,1 & 18,1 & 18,1 & 18,1 \\
Margarina sem sal & 5,7 & 5,7 & 5,7 & 5,7 & 5,7 \\
Essência de baunilha & 1,5 & 1,5 & 1,5 & 1,5 & 1,5 \\
Fermento químico em pó & 0,6 & 0,6 & 0,6 & 0,6 & 0,6 \\
Sal refinado & 0,3 & 0,3 & 0,3 & 0,3 & 0,3 \\
Farinha de casca de batata & 0,0 & 6,0 & 8,5 & 11,0 & 13,5 \\
\hline
\end{tabular}

Para o preparo dos cookies, o chocolate foi cortado em cubos e reservado. Em uma tigela, foram misturados os ovos, o açúcar refinado e a margarina, até completa homogeneização. Em seguida, foram acrescidos a farinha de trigo, a FCB, a essência de baunilha e o sal, misturando-se todos os ingredientes até a mistura ficar homogênea. Finalizado esse processo, o chocolate e o fermento foram adicionados à massa, misturando-se manualmente. Os biscoitos foram moldados em formato cilíndrico (10 cm de diâmetro), sendo dispostos em formas de alumínio retangulares $(33 \mathrm{~cm} \mathrm{x} 15 \mathrm{~cm})$. Em seguida, foram assados em forno pré-aquecido a $180^{\circ} \mathrm{C}$, por 20 minutos. Posteriormente, permaneceram em repouso até atingirem a temperatura ambiente $\left(22^{\circ} \mathrm{C}\right)$, sendo acondicionados em potes plásticos hermeticamente fechados até o momento da análise sensorial.

\subsection{ANÁLISE SENSORIAL}

Os testes foram conduzidos no Laboratório de Análise Sensorial do Departamento de Engenharia de Alimentos da Universidade Estadual do Centro-Oeste (Unicentro), em cabines individuais e com iluminação de cor branca. 
A análise sensorial foi realizada por uma equipe de 62 provadores não treinados, constituída por alunos, funcionários e professores da Unicentro, de ambos os gêneros e com idade entre 18 e 55 anos. Foram avaliados os atributos de aparência, aroma, sabor, textura e cor. As amostras foram analisadas por meio de uma escala hedônica estruturada de nove pontos (1: desgostei muitíssimo a 9: gostei muitíssimo). Foram aplicadas, também, questões de aceitação global com o auxílio de escala hedônica estruturada de nove pontos e intenção de compra utilizando-se uma escala estruturada de cinco pontos (1: certamente não compraria a 5: certamente compraria). ${ }^{9}$ Os provadores receberam uma porção de cada amostra (aproximadamente $15 \mathrm{~g}$ ), em pratos plásticos brancos, codificados com números de três dígitos, de forma balanceada e casualizada, acompanhada de um copo de água para a limpeza do palato. As formulações foram oferecidas de forma monádica sequencial.

Um teste de comparação múltipla foi aplicado para comparar as amostras elaboradas na pesquisa com um cookie comercializado (referência) sem adição de FCB. Cada provador identificou se as formulações apresentavam sabor melhor, igual ou pior ao produto de referência, por meio de uma escala hedônica estruturada variando da nota 1 (extremamente pior que a referência) à nota 9 (extremamente melhor que a referência). ${ }^{9}$

\section{5 ÍNDICE DE ACEITABILIDADE}

O cálculo do Índice de Aceitabilidade (IA) foi realizado conforme a fórmula: IA $(\%)=\mathrm{A} x$ 100/B (onde: $A=$ nota média obtida para o produto e $B=$ nota máxima dada ao produto). ${ }^{9}$

\subsection{ANÁLISE FÍSICO-QUÍMICA}

As seguintes análises físico-químicas foram realizadas em triplicata na FCB, na formulação padrão e naquela com maior nível de adição de FCB e com aceitação sensorial semelhante ao produto padrão:

a) Umidade: foi determinada em estufa a $105^{\circ} \mathrm{C}$ até peso constante; $;^{10}$

b) Cinzas: foram analisadas em mufla $\left(550{ }^{\circ} \mathrm{C}\right) ;{ }^{10}$

c) Lipídios totais: utilizou-se o método de extração a quente com extrator de Soxhlet e éter de petróleo; ${ }^{10}$

d) Proteínas: foram avaliadas mediante o teor de nitrogênio total da amostra, pelo método Kjeldahl, determinado ao nível semimicro. ${ }^{10}$ Utilizou-se o fator de conversão de nitrogênio para proteína de 6,25;

e) Fibra alimentar: o teor de fibra alimentar total foi avaliado por cálculo teórico; ${ }^{11,12}$

f) Carboidratos: a determinação de carboidratos foi realizada por meio de cálculo teórico (por diferença) nos resultados das triplicatas, conforme a fórmula: \% Carboidratos $=100-(\%$ umidade + \% proteína + \% lipídios + \% cinzas + \% fibra alimentar); 
g) Valor calórico total (kcal): foi calculado utilizando os seguintes valores: lipídios $(8,37$ $\mathrm{kcal} / \mathrm{g})$, proteína $(3,87 \mathrm{kcal} / \mathrm{g})$ e carboidratos $(4,11 \mathrm{kcal} / \mathrm{g}) .{ }^{13}$

\subsection{DETERMINAÇÃO DO VALOR DIÁRIO DE REFERÊNCIA (VD)}

O Valor Diário de Referência (VD) foi calculado em relação a $30 \mathrm{~g}$ da amostra, com base nos valores médios diários preconizados para adultos (18 a 55 anos), ${ }_{14}^{14}$ resultando em: 2.276,19 kcal/dia, 288,06 g/dia de carboidratos, 82,22 g/dia de proteínas, 83,66 g/dia de lipídios e 15,40 g/dia de fibra alimentar.

\subsection{ANÁLISE ESTATÍSTICA}

Os dados foram analisados com auxílio do software Statgraphics Plus , versão 5.1, mediante análise de variância (ANOVA). A comparação de médias foi realizada pelo teste de médias de Tukey, t de student e Dunnett, avaliados com nível de 5\% de significância.

\subsection{QUESTÕES ÉTICAS}

Este trabalho foi aprovado pelo Comitê de Ética em Pesquisa da Unicentro, parecer n. 608.950/2014. Como critérios de exclusão foram considerados os seguintes fatores: possuir alergia a algum ingrediente utilizado na elaboração dos cookies ou não entregar o Termo de Consentimento Livre e Esclarecido (TCLE) assinado.

\section{RESULTADOS E DISCUSSÃO}

\subsection{ANÁLISE SENSORIAL}

Os resultados da análise sensorial dos cookies são mostrados na Tabela 2. 
Tabela 2 - Médias do índice de aceitabilidade (IA) e dos testes sensoriais afetivos de aceitação e de intenção de compra das formulações de cookies padrão (F1) e com 6\% (F2), 8,5\% (F3), 11\% (F4) e 13,5\% (F5) de farinha de casca de batata $(\mathrm{FCB})^{\dagger}$

\begin{tabular}{crrrrr}
\hline Formulações/ & \multicolumn{1}{c}{ F1 } & \multicolumn{1}{c}{ F2 } & \multicolumn{1}{c}{ F3 } & \multicolumn{1}{c}{ F4 } & \multicolumn{1}{c}{ F5 } \\
\hline Atributos & Média $\pm E P M$ & Média $\pm E P M$ & Média $\pm E P M$ & Média $\pm E P M$ & Média $\pm E P M$ \\
Aparência & $8,10 \pm 0,13^{\mathrm{a}}$ & $7,32 \pm 0,16^{\mathrm{ab}}$ & $6,93 \pm 0,17^{\mathrm{b}}$ & $5,51 \pm 0,23^{\mathrm{c}}$ & $4,48 \pm 0,26^{\mathrm{d}}$ \\
IA (\%) & 89,88 & 81,33 & 77,00 & 61,22 & 49,77 \\
Aroma & $6,58 \pm 0,22^{\mathrm{a}}$ & $6,40 \pm 0,22^{\mathrm{a}}$ & $6,30 \pm 0,22^{\mathrm{a}}$ & $4,79 \pm 0,27^{\mathrm{b}}$ & $4,08 \pm 0,26^{\mathrm{b}}$ \\
IA (\%) & 73,11 & 71,11 & 70,00 & 53,22 & 45,33 \\
Sabor & $8,20 \pm 0,12^{\mathrm{a}}$ & $7,50 \pm 0,11^{\mathrm{a}}$ & $7,52 \pm 0,10^{\mathrm{a}}$ & $5,79 \pm 0,22^{\mathrm{b}}$ & $4,72 \pm 0,23^{\mathrm{c}}$ \\
IA (\%) & 91,11 & 83,33 & 83,55 & 64,33 & 52,44 \\
Textura & $8,06 \pm 0,13^{\mathrm{a}}$ & $7,15 \pm 0,12^{\mathrm{b}}$ & $7,27 \pm 0,16^{\mathrm{b}}$ & $6,19 \pm 0,21^{\mathrm{c}}$ & $5,19 \pm 0,24^{\mathrm{d}}$ \\
IA (\%) & 89,55 & 79,44 & 80,77 & 68,77 & 57,66 \\
Cor & $8,01 \pm 0,15^{\mathrm{a}}$ & $6,82 \pm 0,14^{\mathrm{b}}$ & $7,27 \pm 0,16^{\mathrm{b}}$ & $5,69 \pm 0,22^{\mathrm{c}}$ & $4,54 \pm 0,23^{\mathrm{d}}$ \\
IA (\%) & 89,00 & 75,77 & 80,77 & 63,22 & 50,44 \\
Aceitação global & $7,64 \pm 0,14^{\mathrm{a}}$ & $6,28 \pm 0,17^{\mathrm{b}}$ & $6,35 \pm 0,18^{\mathrm{b}}$ & $5,45 \pm 0,23^{\mathrm{c}}$ & $4,33 \pm 0,25^{\mathrm{d}}$ \\
IA (\%) & 84,88 & 69,77 & 70,55 & 60,55 & 48,11 \\
Intenção de compra & $4,40 \pm 0,09^{\mathrm{a}}$ & $3,76 \pm 0,11^{\mathrm{b}}$ & $3,86 \pm 0,14^{\mathrm{b}}$ & $2,61 \pm 0,14^{\mathrm{c}}$ & $2,12 \pm 0,13^{\mathrm{c}}$ \\
IA (\%) & 88,00 & 75,20 & 77,20 & 52,20 & 42,40 \\
\hline
\end{tabular}

Maiores notas para aparência foram verificadas para F1 quando comparadas a F3, F4 e F5. Já as amostras F2 e F3 apresentaram maiores notas que F4 e F5, enquanto F4 teve maiores notas que F5 ( $<<0,05)$. Contudo, F2 e F3 não demonstraram diferença significativa entre si ( $>>0,05)$. Resultados similares foram verificados por Fernandes, ${ }^{15}$ que avaliou a aceitação de pães integrais adicionados de $\operatorname{FCB}\left(3,6,9\right.$ e 12\%). A FCB apresenta menor luminosidade que a farinha de trigo, ${ }^{16}$ fato que pode explicar as menores notas para aparência dos cookies.

Verificaram-se maiores notas para F1, F2 e F3 comparando-se a F4 e F5 (p<0,05) no atributo aroma. Efeitos semelhantes foram constatados para o sabor, em que maiores notas permaneceram para F1, F2 e F3 comparando-se a F4 e F5; porém, F4 teve maior aceitação que F5 (p<0,05), corroborando a literatura. ${ }^{15}$ As menores notas verificadas para sabor e aroma para os produtos com maior adição de FCB podem ser explicadas em razão da presença de compostos voláteis presentes na casca de batata, como os alcoóis alifáticos e aldeídos, os quais podem alterar as características sensoriais dos produtos. ${ }^{17}$

$\mathrm{Na}$ avaliação dos atributos textura, cor e aceitação global, foram obtidas maiores notas para F1 em relação às demais amostras. Também, F2 e F3 tiveram maior aceitação que F4 e F5, e F4 foi melhor aceita que F5 $(\mathrm{p}<0,05)$. De forma semelhante, na intenção de compra, foi constatada que F1 teve as maiores notas entre as amostras $(\mathrm{p}<0,05)$, enquanto F2 e F3 apresentaram maior aceitação que F4 e F5. As amostras F2 e F3, bem como F4 e F5, não demonstraram diferença estatística entre si. Resultados similares foram observados em pesquisa que avaliou pães de forma com adição de farinha de casca de mandioca $(7,5,15,22,5$ e $30 \%) .{ }^{18}$

\footnotetext{
${ }^{\dagger}$ Letras diferentes na linha indicam diferença significativa pelo teste de Tukey $(\mathrm{p}<0,05)$; EPM: erro padrão da média
} 
Durante a elaboração dos cookies foi possível verificar que as amostras com maiores teores de FCB apresentavam-se mais rígidas, fato que acontece em decorrência do enfraquecimento do glúten presente na farinha de trigo, a qual foi reduzida nas formulações. Outra alteração tecnológica foi em relação à cor dos cookies, que ficaram mais escuros após a adição de FCB. Esse fato ocorre em razão da elevada quantidade de fibras presente na casca de batata, ${ }^{19}$ que promove uma coloração mais escura aos produtos, corroborando a literatura. ${ }^{15}$

Em relação ao IA, apenas as formulações F1, F2 e F3 apresentaram valores maiores que 70\% na maioria das avaliações, o que classifica os produtos com boa aceitabilidade. ${ }^{9}$ Resultados similares foram verificados em pesquisa que avaliou a aceitação de biscoitos com adição de FCB (5, 10 e $20 \%){ }^{20}$

Na Figura 1, apresenta-se a distribuição dos provadores pelos valores hedônicos avaliados no teste sensorial.
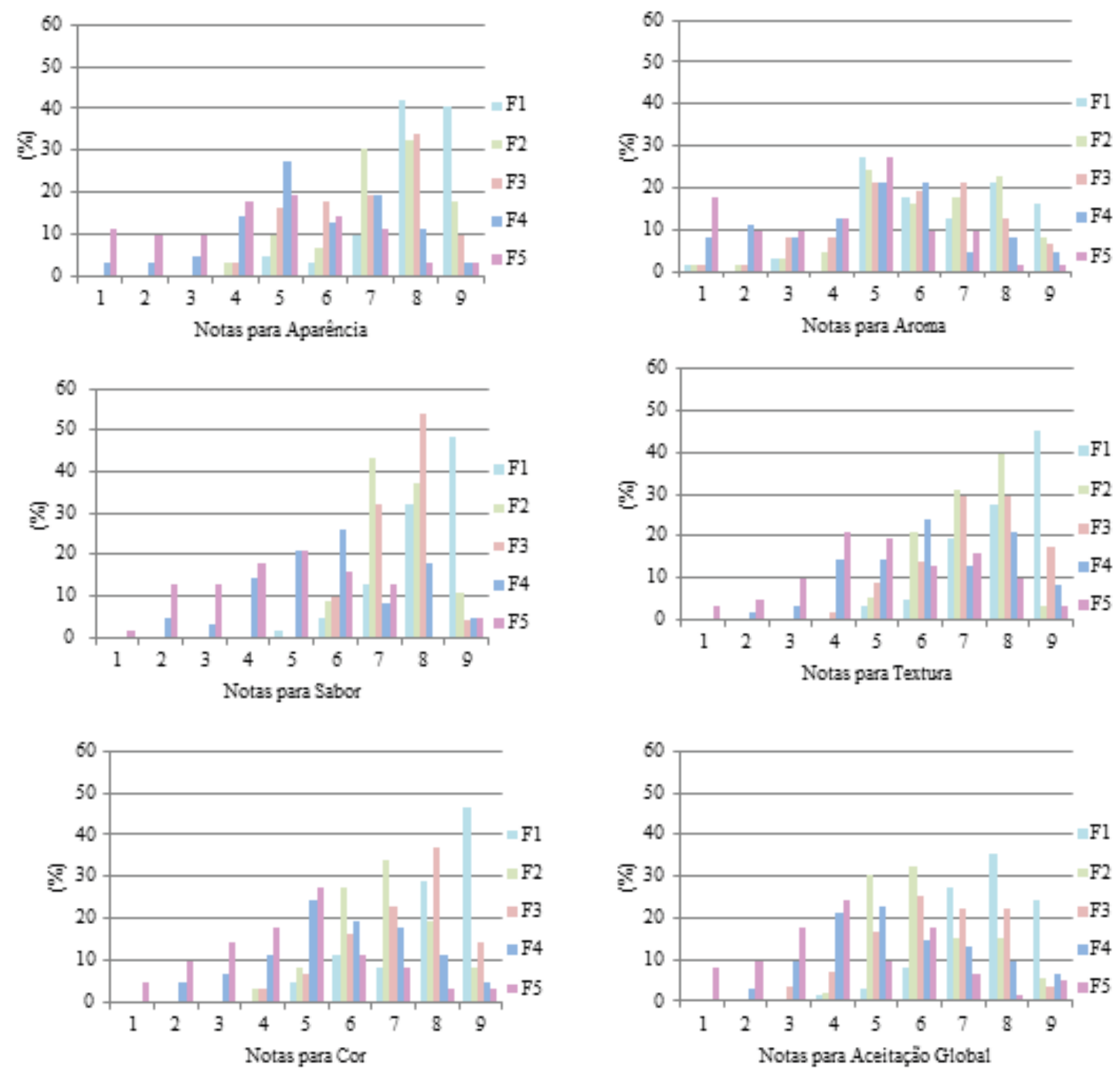

Figura 1 - Distribuição dos provadores pelos valores hedônicos obtidos na avaliação dos atributos aparência, aroma, sabor, textura, cor e aceitação global do cookie padrão (F1) e com 6\% (F2), 8,5\% (F3), 11\% (F4) e 13,5\% (F5) de FCB 
As notas $\geq 7$ (gostei moderadamente) foram as mais referidas pelos provadores, principalmente para os atributos de aparência, sabor, textura e cor. As amostras F4 e F5 tiveram a maior frequência de notas abaixo de 5 (nem gostei, nem desgostei), demonstrando menor aceitação dos cookies com maiores teores de FCB, o que também foi constatado por outros pesquisadores. ${ }^{18}$

Os resultados do teste de comparação múltipla dos cookies são informados na Figura 2.

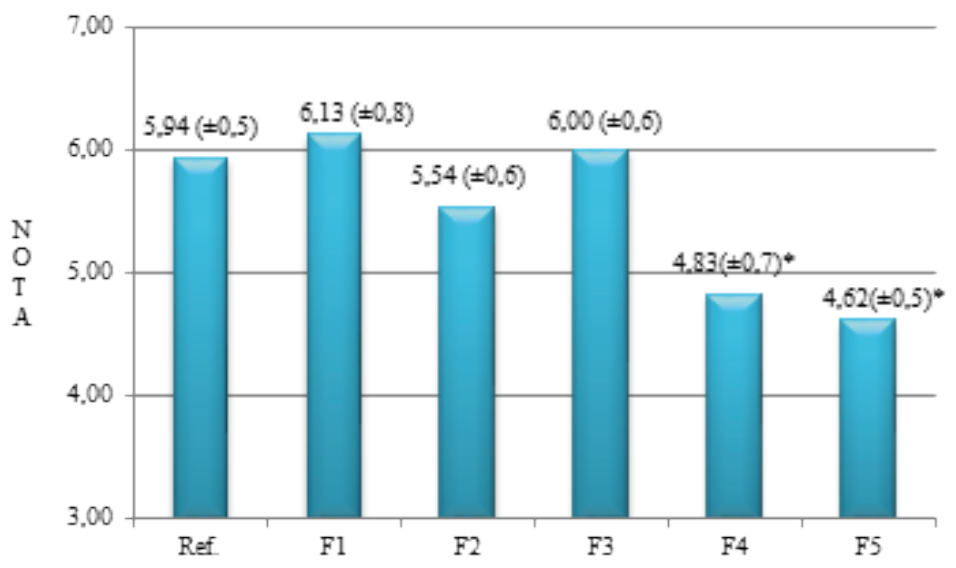

Figura 2 - Notas médias ( \pm desvio padrão) do teste de comparação múltipla das formulações de cookie padrão (F1) e com 6\% (F2), 8,5\% (F3), 11\% (F4) e 13,5\% (F5) de FCB comparadas com um cookie tradicional comercializado (referência) em relação ao sabor

Nota: ${ }^{*}$ diferença significativa do produto referência (teste de Dunnett, $\mathrm{p}<0,05$ ).

Não houve diferença significativa ( $>>0,05)$ entre as amostras F1, F2 e F3 e a marca comercializada, sendo consideradas com sabor semelhante. Entretanto, F4 e F5 foram classificadas com sabor inferior, corroborando a literatura. ${ }^{18}$ Assim, verifica-se a possibilidade de comercialização dos cookies adicionados de até 8,5\% de FCB. Nesse aspecto, a utilização de ingredientes alternativos em produtos alimentícios torna-se viável, já que são bem aceitos pelos consumidores. Além disso, pode-se reduzir os custos de produção e o descarte de resíduos no meio ambiente e melhorar as características nutricionais dos produtos elaborados.

Características relacionadas ao aroma e ao sabor parecem ser as mais importantes na avaliação sensorial de produtos alimentícios. ${ }^{21}$ Em razão disso, a amostra F3 (8,5\% de FCB) foi selecionada para fins de comparação com a padrão (F1), por ser aquela com o maior teor de FCB e com aceitação semelhante à formulação padrão nessas duas características.

\subsection{COMPOSIÇÃO FÍSICO-QUÍMICA}

A composição físico-química da FCB, do cookie padrão e daquele com adição de $8,5 \%$ de FCB está descrita na Tabela 3. 
Tabela 3 - Composição físico-química e valores diários recomendados - VD* (porção média de 30 gramas - 6 unidades) da farinha de casca de batata (FCB), do cookie padrão (F1) e daquele adicionado de 8,5\% de FCB (F3) ${ }^{*}$

\begin{tabular}{|c|c|c|c|c|c|}
\hline & FCB & \multicolumn{2}{|c|}{ F1 } & \multicolumn{2}{|c|}{ F3 } \\
\hline Avaliação & Média $\pm D P$ & Média $\pm D P$ & $\operatorname{VD}(\%) 1^{\varsigma}$ & Média $\pm D P$ & $\operatorname{VD}(\%)^{\S}$ \\
\hline Umidade (\%) & $5,47 \pm 0,04$ & $12,16 \pm 0,09^{a}$ & ND & $12,57 \pm 0,03^{a}$ & ND \\
\hline Cinzas $\left(\mathrm{g} .100 \mathrm{~g}^{-1}\right)$ & $5,57 \pm 0,02$ & $1,43 \pm 0,05^{\mathrm{b}}$ & ND & $1,86 \pm 0,05^{\mathrm{a}}$ & ND \\
\hline $\begin{array}{l}\text { Proteínas } \\
\left(\text { g. } 100 \mathrm{~g}^{-1}\right)\end{array}$ & $11,51 \pm 0,04$ & $9,79 \pm 0,08^{b}$ & 3,56 & $10,36 \pm 0,07^{a}$ & 3,77 \\
\hline $\begin{array}{l}\text { Lipídios } \\
\left(\text { g. } 100 \mathrm{~g}^{-1}\right)\end{array}$ & $0,30 \pm 0,05$ & $11,54 \pm 0,07^{a}$ & 4,13 & $10,22 \pm 0,08^{\mathrm{b}}$ & 3,65 \\
\hline $\begin{array}{l}\text { Carboidratos } \\
\left(\text { g. } 100 \mathrm{~g}^{-1}\right)\end{array}$ & $77,15 \pm 0,08$ & $65,07 \pm 0,07^{\mathrm{a}}$ & 6,77 & $64,99 \pm 0,07^{a}$ & 6,76 \\
\hline $\begin{array}{l}\text { Calorias } \\
\left(\mathrm{kcal} 100 \mathrm{~g}^{-1}\right)\end{array}$ & $364,16 \pm 0,25$ & $401,96 \pm 0,33^{a}$ & 5,29 & $392,74 \pm 0,29^{a}$ & 5,17 \\
\hline $\begin{array}{l}\text { Fibra alimentar } \\
(\text { g. } 100 \mathrm{~g}-1) 2 \|\end{array}$ & 26,60 & 1,70 & 11,03 & 3,96 & 25,71 \\
\hline
\end{tabular}

Fernandes, 15 avaliando casca de batata, verificou valores inferiores ao presente estudo para carboidratos $(61,30 \%)$, cinzas $(2,78 \%)$ e calorias $(351,59 \%)$, porém superiores para umidade $(9,72 \%)$, proteína $(12,97 \%)$ e lipídios (2,45\%). Esses resultados podem ser explicados por diferenças no tempo de secagem da farinha, regiões de plantio e condições genéticas da planta, entre outras. ${ }^{22}$ A FCB apresentou teor de umidade conforme o valor preconizado pela legislação brasileira para farinhas (máximo de $15 \%$ ). ${ }^{23}$

Não houve diferença entre os conteúdos de umidade, carboidratos e calorias para F1 e F3 $(\mathrm{p}>0,05)$. Contudo, maiores teores de cinzas e proteínas e menores de lipídios foram verificados em F3, em razão de que a farinha de trigo apresenta menores teores de cinzas $(0,8 \mathrm{~g} \cdot 100 \mathrm{~g}-1)$ e proteínas (9,8 g.100g-1) e maiores de lipídios (1,4 g.100g-1) que a FCB (Tabela 3).12 Esses resultados tornam os cookies com FCB uma opção alimentar mais saudável, já que um menor consumo de lipídios pode reduzir o risco do desenvolvimento de doenças cardiovasculares, dislipidemias e obesidade. ${ }^{24}$

Destaca-se como principal resultado deste trabalho o teor de fibras verificado na formulação adicionada de 8,5\% de FCB (F3), expressando um aumento significativo de 132,94\% em relação a F1. Esse efeito decorre do elevado teor de fibras da FCB (26,6 g.100g-1),11 muito superior ao encontrado na farinha de trigo comum (2,3 g.100g-1). ${ }^{12}$ Nesse aspecto, a amostra F3 pode ser considerada um produto fonte de fibra alimentar, já que possui um teor mínimo de $3 \%$ de fibras em sua composição. ${ }^{25}$

\section{CONCLUSÃO}

Um nível de adição de até $8,5 \%$ de FCB em cookies foi bem aceito pelos provadores. Além disso, proporcionou um aumento no conteúdo de cinzas, proteínas e fibras e reduziu os teores de lipídios, melhorando o perfil nutricional do produto. Assim, a FCB pode ser considerada um potencial ingrediente para adição em cookies e similares, os quais podem ser oferecidos aos consumidores com altas expectativas de aceitação no mercado. 


\section{REFERÊNCIAS}

1. Empresa Brasileira de Pesquisa Agropecuária (Embrapa). Desperdício de alimentos é desperdício de recursos naturais e financeiros [Internet]. Brasília, DF; 2013 [cited 2017 abr 10]. Disponível em: https://www.embrapa.br/busca-de-noticias/-/noticia/1493220/desperdicio-de-alimentos-e-desperdicio-de-recursos-naturais-e-financeiros

2. Storck CR, Nunesi GL, Oliveira BB, Basso C. Folhas, talos, cascas e sementes de vegetais: composição nutricional, aproveitamento na alimentação e análise sensorial de preparações. Ciên Rural 2013;43(3):537-43.

3. Instituto Brasileiro de Geografia e Estatística (IBGE). Indicadores IBGE - Estatística da Produção Agrícola [Internet]. 2016 [acesso em 2017 abr 22]. Disponível em: http://www.ibge.gov.br/ home/estatistica/indicadores/agropecuaria/lspa/

4. Souza AM, Pereira RA, Yokoo EM, Levy RB, Sihieri R. Alimentos mais consumidos no Brasil: inquérito Nacional de Alimentação 2008-2009. Rev. Saúde Púb. 2013;47(1):190-9.

5. Singh PP, Saldaña MDA. Subcritical water extraction of phenolic compounds from potato peel. Food Res Int. 2011;44(8):2452-8.

6. Miranda AA, Caixeta ACA, Flávio EF, Pinho L. Desenvolvimento e análise de bolos enriquecidos com farinha da casca do maracujá (Passiflora edulis) como fonte de fibras. Alim Nut. 2012;24(2):225-32.

7. Associação Brasileira das Indústrias de Biscoitos, Massas Alimentícias e Pães \& Bolos Industrializados (ABIMAPI). Categorias de biscoitos, massas alimentícias, pães e bolos industrializados fecham 2015 com faturamento de R\$ 35,4 bilhões [Internet]. 2016. [acesso em 2017 jun 02]. Disponível em: https://www.abimapi.com.br/noticias-detalhe.php?i=MTc1OQ==

8. Rossini K, Anzanello MJ, Fogliatto FS. Data mining-based method for identifying discriminant attributes in sensory profiling. Food Qual Pref. 2011;22(1):139-48.

9. Dutcosky SD. Análise sensorial de alimentos. 3a ed. Curitiba: Champagnat; 2011.

10. Association of Official Analytical Chemistry (AOAC). Official Methods of Analysis of AOAC International. 18th ed. 4 rev. Gaithersburg: AOAC; 2011.

11. Camire ME, Violette D, Dougherty MP, Mclaughlin MA. Potato peel dietary fiber composition: effects of peeling and extrusion cooking processes. J Agric Food Chem. 1997;45(4):1404-8.

12. Tabela Brasileira de Composição dos Alimentos (TACO). 4a ed. rev. e ampl. Campinas: NEPA; 2011.

13. Merril AL, Watt BK. Energy values of foods: basis and derivation. Washington: United States Department of Agriculture Handbook; 1973. 
14. Dietary Reference Intakes (DRI). Dietary Reference Intakes for energy, carbohydrate, fiber, fat, fatty acids, cholesterol, protein and amino acids. Washington: The National Academies Press; 2005.

15. Fernandes AF. Utilização de farinha de casca de batata (solanum tuberosum L.) na elaboração de pão integral [dissertação]. Lavras: Universidade Federal de Lavras; 2006.

16. Fernandes AF, Pereira J, Germani R, Oiano-Neto J. Efeito da substituição parcial da farinha de trigo por farinha de casca de batata (Solanum Tuberosum Lineu). Ciênc. Tecnol. Aliment. 2008;28(1):56-65.

17. Oruna-Concha MJ, Bakker J, Ames JM. Comparison of the volatile components of two cultivars of potato cooked by boiling, conventional baking and microwave baking. J Sci Food Agric. 2002;82(9):1080-7.

18. Vilhalva DAA, Soares Júnior MS, Mouro CMA, Caliari M, Souza TAC, Silva FA. Aproveitamento da farinha de casca de mandioca na elaboração de pão de forma. Rev. Inst. Adolfo Lutz 2011;70(4):514-21.

19. Arora A, Camire ME. Performance of potato peels in muffins and cookies. Food Res. Int. 1994;27(1):15-22.

20. Garmus TT, Bezerra JRM, Rigo M, Córdova KRV. Elaboração de biscoito com adição de farinha de casca de batata (Solanum Tuberosum L.). Rev. Bras. Tecnol. Agroin. 2009;3(2):56-65.

21. Behrens JH, Silva MAAP, Wakeling IN. Avaliação da aceitação de vinhos brancos varietais brasileiros através de testes sensoriais afetivos e técnicas multivariadas de mapa de preferência interna. Ciênc. Tecnol. Alimen. 1999;19(2):214-20.

22. Rodriguez-Sandoval E, Sandoval G, Cortes-Rodriguez M. Effect of quinoa an potato flours on the thermomechanical and breadmaking properties of wheat flour. J Braz Chem. 2012;29(3):503-10.

23. Brasil. Agência Nacional de Vigilância Sanitária. RDC n. 263, de 22 de setembro de 2005. Regulamento técnico para produtos de cereais, amidos, farinhas e farelos. Diário Oficial [da] União. Brasília: Poder Executivo; 2005.

24. Cervato AM, Mazzilli RN, Martins IS, Marucci MFN. Dieta habitual e fatores de risco para doença cardiovasculares. Rev. Saúde Púb. 1997;31(3):227-35.

25. Brasil. Agência Nacional de Vigilância Sanitária. RDC n. 54, de 12 de novembro de 2012. Regulamento Técnico sobre Informação Nutricional. Diário Oficial [da] União. Brasília: Poder Executivo; 2012.

Data da submissão: 24 de abril de 2017 Avaliado em: 31 de maio de 2017 (AVALIADOR A) Avaliado em: 11 de maio de 2017 (AVALIADOR B) Aceito em: 02 de junho de 2017 